\title{
Latido de vida: vivencias del paciente con enfermedad cardiovascular en una unidad de cuidado intensivo
}

\section{Life heartbeat: experiences of patients with cardiovascular disease in an intensive care unit Batimento pela vida: vivências do paciente com doença cardiovascular na unidade de terapia intensiva}

\author{
Linda Marcela Peña-Ortiz ${ }^{*}$ \\ Yulitza Andrea Hernández-Moncada** \\ Johan Manuel Morales-Morales ${ }^{* * *}$ \\ Gloria Zambrano-Plata*** \\ Claudia Sánchez $z^{* * * * *}$
}

\begin{abstract}
Autor de correspondencia
* $\triangle$ Estudiante de Enfermería VII semestre, Universidad Francisco de Paula Santander. Correo: linm_0815@hotmail.com. Orcid: https://orcid org/0000-0003-3030-3734. San José de Cúcuta, Norte de Santander, Colombia

** Estudiante de Enfermería VII semestre, Universidad Francisco de Paula Santander. Correo: yulitza andrehernandez@hotmail.com. Orcid: https:/ orcid.org/0000-0003-3760-4361. San José de Cúcuta, Norte de Santander, Colombia.

*** Estudiante de Enfermería VII semestre, Universidad Francisco de Paula Santander. Correo: jese. morales.93@hotmail.com. Orcid: https://orcid. org/0000-0002-5475-9552. San José de Cúcuta, Norte de Santander, Colombia

***** Enfermera, PhD. Docente Facultad Ciencias de la Salud, Universidad Francisco de Paula Santander. Correo: gloriaesperanzazp@ufps.edu.co. Orcid: http://orcid.org/0000-0001-5524-3200. San José de Cúcuta, Norte de Santander, Colombia

******* Especialista de cuidado critico en el adulto. Clínica San José. Enfermera cuidado critico coronario. Correo: cpcp1981@hotmail.com. Orcid: https://orcid.org/0000-0001-5964-2874. San José de Cúcuta, Norte de Santander, Colombia.
\end{abstract}

Este es un artículo bajo la licencia CC BY

(https://creativecommons.org/

licenses/by/4.0/) @) ()

\section{Resumen}

Objetivo: Conocer las vivencias del paciente con enfermedad cardiovascular durante su estancia en la unidad coronaria de cuidados intensivos, en una clínica de cuarto nivel del Norte de Santander, en el año 2017. Materiales y Métodos: Estudio cualitativo con enfoque fenomenológico; se usó la entrevista directa a profundidad para la recolección de datos. Los casos estudiados fueron seis pacientes que presentaron una enfermedad cardiovascular y que tuvieron una estancia mayor o igual a tres días en la unidad coronaria, quienes participaron de manera voluntaria y con residencia en la ciudad de Cúcuta. Resultados: Las vivencias al sufrir una enfermedad cardiovascular y su hospitalización en la Unidad de Cuidados Intensivos, se dilucidó a través del tema central: Estancia en UCI como experiencia renovadora. Emergieron seis categorías: atención con calidad humana; incertidumbre ante la enfermedad; impacto ante una situación inesperada; Fe en Dios; familia, como pilar fundamental; y pérdida de la autonomía considerada como factor estresante. Conclusiones: Para el paciente con enfermedad cardiovascular, la hospitalización en la Unidad de Cuidados Intensivos es una vivencia muy estresante y dolorosa. Dicha experiencia se caracteriza por el temor, la ansiedad y la incertidumbre en relación con los efectos de la enfermedad, los equipos utilizados en el tratamiento, los procedimientos terapéuticos y los cuidados de bienestar y confort. Estas vivencias están matizadas por aspectos positivos derivados de la atención con calidad humana del personal de salud y del apoyo familiar, factores que fortalecen al paciente; así como de la fe en Dios que le da trascendencia a esta experiencia de vida.

Palabras Clave: Investigación cualitativa, UCI, experiencias, enfermería, enfermedades cardiovasculares. 


\title{
Resumo
}

Objetivo: Conhecer as vivencias do paciente com doença cardiovascular durante a sua permanência na unidade coronariana de terapia intensiva numa clínica da rede privada do estado de Norte de Santander no ano de 2017. Materiais e métodos: Estudo qualitativo de tipo fenomenológico. Usou-se a entrevista direta à profundidade para a coleta dos dados. Foram estudados 6 pacientes residentes da cidade de Cúcuta com diagnóstico de doença cardiovascular com uma permanência hospitalar maior ou igual de 3 dias. Resultados: As vivências de padecer uma doença cardiovascular e a sua internação na Unidade de Terapia Intensiva (UTI) evidenciou-se pela temática principal: Permanência na UTI como experiência renovadora. Surgiram seis categorias: atenção com qualidade humana, insegurança e dúvida perante a doença, impacto perante a uma situação inesperada, fé em Deus, família como eixo fundamental e perdida da autonomia sendo considerada esta última um fator estressante. Conclusões: Para o paciente com doença cardiovascular a internação na UTI é uma vivencia estressante e dolorida. É uma experiência caracterizada por temor, ansiedade e duvidas relacionadas com o desfecho da doença, dos equipamentos usados no tratamento, os procedimentos terapêuticos e os cuidados para o bem-estar e conforto. Essas vivências são melhoradas com aspectos positivos da atenção com qualidade humana do pessoal de saúde e pelo apoio familiar. Fatores que fortalecem o doente são a fé em Deus que dá transcendência a experiência vivida.

Palavras-chave: Pesquisa qualitativa, unidades de terapia intensiva, experiências, enfermagem, doenças cardiovasculares.

\begin{abstract}
Objective: Know the experiences of patients with cardiovascular disease during their stay at the coronary care unit in a fourth level clinic in Norte de Santander (Colombia), in 2017. Materials and Methods: Qualitative study with a phenomenological focus; a depth, direct interview was used for the recollection of data. The studied cases were six patients that presented cardiovascular disease and stayed three or more days at the coronary unit, the patients participated voluntarily and reside in the city of Cucuta. Results: The experiences when suffering from a cardiovascular disease and being hospitalized in the Intensive Care Unit were clarified through the central subject: Stay at the ICU as a renewing experience. Six categories emerged: human quality care; uncertainty regarding the disease; reaction towards an unexpected situation; faith in God; family as fundamental pillar; and loss of autonomy, considered a stressing factor. Conclusions: For the patient with cardiovascular disease, hospitalization in an Intensive Care Unit is a stressful and painful experience. Such experience is characterized by fear, anxiety and uncertainty relating to the effects of the disease, the equipment used in the treatment, the therapeutic procedures, and welfare and comfort care. These experiences are nuanced by positive aspects derived from care with human quality from the health personnel and family support, factors that give the patient strength; as well as the faith in God, which gives them transcendence in this life experience.
\end{abstract}

Keywords: Qualitative research, ICU, experiences, nursing, cardiovascular diseases.

\section{Introducción}

Las enfermedades cardiovasculares (ECV) son un grupo de desórdenes del corazón y de los vasos sanguíneos, conforman este grupo: la aterosclerosis, enfermedad cardíaca coronaria, angina de pecho, infarto agudo de miocardio, enfermedad cerebro vascular, hipertensión arterial, arteriopatías periféricas, cardiopatías reumáticas, cardiopatías congénitas, trombosis venosas profundas, embolias pulmonares e insuficiencia cardíaca (1).

Según la Organización Mundial de la Salud (OMS) cada año mueren más personas por ECV que por cualquier otra causa. Se calcula que para el año 2015 murieron por esta causa 17,7 millones de personas, lo cual representa un $31 \%$ de todas las muertes registradas en el mundo (1). En Estados Unidos, aproximadamente 80 millones de adultos sufren de algún tipo de enfermedad cardiovascular, es por esta razón que estas enfermedades son las responsables del $42 \%$ de todas las muertes cada año en dicho país (2-3).

Actualmente, en Colombia las enfermedades del sistema circulatorio fueron la primera causa de mortalidad en hombres y en mujeres, entre los años 2005 y 
ISSN-PRINT

1794-9831

E-ISSN 2322-7028

Vol. 16 No. 2

May - Ago 2019

Cúcuta, Colombia
2014, lo que corresponde a 78,24 muertes por cada 100.000 habitantes (4-5).

Cifras de El Departamento Administrativo Nacional de Estadística (DANE) para Norte de Santander, evidencian la relación de las altas cifras de mortalidad como causa del padecimiento de enfermedades cardiovasculares en la región, indicando que se han presentado 186 muertes por cada 100.000 habitantes entre los años 2005 y 2014 (6).

La gravedad de la enfermedad coronaria obliga en numerosas ocasiones al ingreso de pacientes afectados por esta patología a unidades especiales de cuidados intensivos donde se procede a tratar la enfermedad. Para un paciente que está hospitalizado en la Unidad de Cuidados Intensivos (UCI) es un suceso desconocido para él, generando diferentes emociones y sentimientos (7). "El paciente es arrancado de su entorno, de su hogar, separado de sus seres queridos, sometido a numerosas técnicas invasivas dolorosas y siente que la muerte puede estar cercana" (8).

La vivencia de estar hospitalizado en la Unidad de Cuidado Intensivo (UCI) crea una de las mayores crisis en la persona que pasa por este proceso. La literatura documenta que este tipo de hospitalización genera en el individuo alteraciones psicológicas como ansiedad, angustia y estrés (9-10).

Por otra parte, al considerarse la enfermedad como un proceso patológico, hace que la persona experimente una serie de cambios relacionados con: pérdidas de rol, de status, de función o parte corporal, alteración de la autoimagen y del autoconcepto, pérdida de relaciones y lazos afectivos e incluso el individuo establece una relación estrecha con su propia muerte (7-11).

Las consecuencias relacionadas con las patologías anteriores hacen que los pacientes experimenten: separación de su entorno, pérdida del contacto familiar, aislamiento, dolor por múltiples procedimientos invasivos, ruido de los equipos que genera angustia, el ambiente frío, etc. Estos factores generan en el paciente una situación de miedo e incertidumbre con el entorno hospitalario (12-13).

Diversos artículos mencionan que los pacientes recuerdan bastante bien sus vivencias durante su estancia en la UCI, a veces en detalles vívidos (8, 14-15).
Los pacientes rememoran no sólo las vivencias negativas sino también las neutras e incluso las positivas. Las vivencias positivas incluyen un sentido de seguridad promovido especialmente por las enfermeras. Las vivencias negativas incluyen deterioro del funcionamiento cognitivo y molestias tales como problemas relacionados con el sueño, el dolor y la ansiedad (16).

Si no se conoce la vivencia de una persona que está en la unidad de cuidados intensivos, desde el punto de vista del profesional de enfermería, corresponde a lo planteado por Madjar al asegurar "que los registros que los enfermeros elaboran contienen descripciones de la experiencia observada, no vivida, en las cuales se consignan procedimientos realizados y las respuestas desde el punto de vista físico y en ocasiones psicológicas" (17), lo que significa que no se podría tener una percepción completa de la vivencia por la que está pasando el paciente con el solo hecho de verlo, y por tanto no entender la magnitud de algo tan complejo, como lo es el estar críticamente enfermo.

Este es el punto que da partida de inicio a la presente investigación, por el cual surge la necesidad de conocer las vivencias de las personas ante un evento como lo es su estancia en la UCI debido a una enfermedad cardiaca. Desde esta perspectiva se realizará una aproximación cercana al mundo subjetivo del paciente con relación a su estado de salud, ya sea emocional o fisiológico. Es por eso, que interesa conocer los diferentes sentimientos y pensamientos respecto a esta circunstancia de la vida. Igualmente, se pretenden obtener nuevas pautas, estableciendo acciones y actuaciones por parte de la enfermería dirigidas a minimizar los sentimientos negativos, con el objetivo de alcanzar una evolución más satisfactoria de la enfermedad. Teniendo en cuenta en que no se han hecho investigaciones sobre este tema hasta la fecha a nivel regional, con este estudio se pretende profundizar sobre la forma como se debe tratar en todos los aspectos el paciente con enfermedad cardiovascular.

Se aplicaron diversas teóricas como el cuidado humanizado de Jean Watson, la de la incertidumbre de Merle Mishel y la psicodinámica de enfermería, que se basa en las relaciones interpersonales enfermeropaciente de Hildegard Peplau, entre otras. Estas teorías permitieron determinar la importancia de la investigación, en la que se debe profundizar sobre la evidencia relacionada con el actuar de la enfermería, que debe ser individualizada y según las necesidades 
de cada paciente, con la mirada puesta más allá del aspecto biológico, de modo que englobe un tratamiento más holístico.

\section{Objetivo}

Conocer las vivencias del paciente con enfermedad cardiovascular, durante su estancia en la unidad coronaria de cuidados intensivos, en una clínica de cuarto nivel de Norte de Santander, durante el primer semestre de 2017.

\section{Materiales y métodos}

Para el análisis de los datos se utilizó la metodología cualitativa con un enfoque fenomenológico descriptivo, basado en el planteamiento filosófico de Edmund Husserl. Esta fenomenología, permite describir las experiencias de vida del paciente tal como se presentan en su conciencia desde sus subjetividades.

Para la recolección de datos se realizaron doce entrevistas a profundidad semi-estructuradas, dos entrevistas por cada participante, alcanzando una saturación de los datos (18-19). En promedio cada entrevista tuvo una duración de 45 minutos, entre la primera y segunda hubo un intervalo de cuatro a cinco días. Todas las entrevistas fueron grabadas con el consentimiento de los participantes.

Igualmente, se realizó un muestreo intencional, en el que participaron seis sujetos con enfermedad cardiovascular, que requirieron hospitalización en la UCI coronaria, por agudización de su enfermedad de base. Todos estos pacientes presentaron una estancia mayor o igual a tres días.
En cuanto al método de análisis de la información obtenida se realizaron los siguientes pasos mencionados por Ulin et al (20). - Lectura: establecer una relación íntima con los datos. - Codificación: identificar los temas que surgen. - Presentación de los datos: distinción de los matices de un tema. - La reducción de los datos: llegar al panorama general. - La interpretación. - Síntesis de los resultados: lagunas y conexiones (21).

Con el fin de garantizar el rigor y la calidad científica de la investigación se tuvieron en cuenta los siguientes criterios: a) credibilidad, para esto los sujetos confirmaron que los descrito en los resultados por los investigadores reflejaba lo expresado por ellos; $b$ ) auditabilidad, en este caso se dispone de archivos de las transcripciones y de los audios de las entrevistas realizadas; y, c) transferibilidad, trata de examinar qué tanto se aplican los resultados a otros contextos (21-22).

Esta investigación se realizó bajo los principios descritos en el informe Belmont de 1978, que formula los principios éticos biomédicos y directrices para la protección en sujetos humanos que sean objeto de investigación. También se dispuso de la resolución $\mathrm{N}^{\circ}$ 8430 de 1993, en su artículo 15, del Ministerio de Salud de Colombia, en donde se menciona que se debe obtener el consentimiento informado de las personas de forma escrita o verbal, lo cual se realizó con cada participante de manera verbal (23). La propuesta investigativa, junto con sus aspectos éticos, fue evaluada y aprobada por el comité curricular del programa de Enfermería de la Universidad Francisco de Paula Santander 
ISSN-PRINT

1794-9831

E-ISSN 2322-7028

Vol. 16 No. 2

May - Ago 2019

Cúcuta, Colombia

\section{Resultados}

Tabla 1. Características sociodemográficas de los pacientes cardiovasculares en la unidad de cuidados intensivos coronaria.

\begin{tabular}{|c|c|}
\hline CARACTERISTICA & $\begin{array}{l}\text { PACIENTES CON ENFERMEDAD CARDIOVAS- } \\
\text { CULAR N }=6\end{array}$ \\
\hline \multicolumn{2}{|c|}{ EDAD (AÑOS) } \\
\hline $50-60$ & 4 \\
\hline $60-70$ & 1 \\
\hline $70-80$ & 1 \\
\hline \multicolumn{2}{|c|}{ SEXO } \\
\hline Mujeres & 2 \\
\hline Hombres & 4 \\
\hline \multicolumn{2}{|c|}{ EDUCACION } \\
\hline \multicolumn{2}{|l|}{ Sin educación } \\
\hline \multicolumn{2}{|l|}{ Primaria incompleta } \\
\hline Primaria & 4 \\
\hline \multicolumn{2}{|l|}{ Secundaria completa } \\
\hline Secundaria & 2 \\
\hline \multicolumn{2}{|c|}{ OCUPACION } \\
\hline Ama de casa & 2 \\
\hline Comerciante & 2 \\
\hline Obrero & 1 \\
\hline Empleado & 1 \\
\hline \multicolumn{2}{|c|}{ ESTRATO SOCIOECONOMICO } \\
\hline $1-2$ & 5 \\
\hline $3-4$ & 1 \\
\hline
\end{tabular}

Fuente: Proceso de recolección de datos, investigación vivencias del paciente cardiovascular durante la estancia en la unidad coronaria 2017.

El proyecto se ejecutó entre los meses de mayo julio de 2017, encontrándose los siguientes resultados:

En la tabla 1 se indican las características de los 6 sujetos con enfermedad cardiovascular participantes del estudio, que estuvieron hospitalizados en la unidad de cuidados intensivos coronaria de una clínica de cuarto nivel. La edad de los pacientes oscilaba entre 50 y 60 años en su mayoría; el tiempo de estancia fue mayor o igual a 3 días.

Por otra parte, en cuanto a las características sociodemográficas de los sujetos participantes en el estudio, en su mayoría corresponden a hombres con un grado de escolaridad entre primaria y secundaria, gran parte labora como comerciantes. Respecto a las dos mujeres participantes, ambas se desempeñan como amas de casa, con nivel de escolaridad de primaria. El estrato socioeconómico de la mayor parte de los participantes estuvo en el rango de 1 y 2 .

Mediante el análisis de los datos obtenidos en las entrevistas, emergió como tema central "Estancia en UCI: experiencia renovadora", y como categorías generales: "Atención con calidad humana", "Incertidumbre ante la enfermedad", "Impacto ante una situación inesperada", "Fe en Dios", "Familia: pilar 
fundamental en la recuperación" y "Perdida de la autonomía un factor estresante".

Conforme a lo descrito anteriormente, estas catego- rías reflejan las vivencias del paciente con enfermedad cardiovascular durante su estancia en la unidad coronaria. En la Figura 1 se visualiza la relación de estas categorías.
E-ISSN 2322-7028

Vol. 16 No. 2

May - Ago 2019

Cúcuta, Colombia

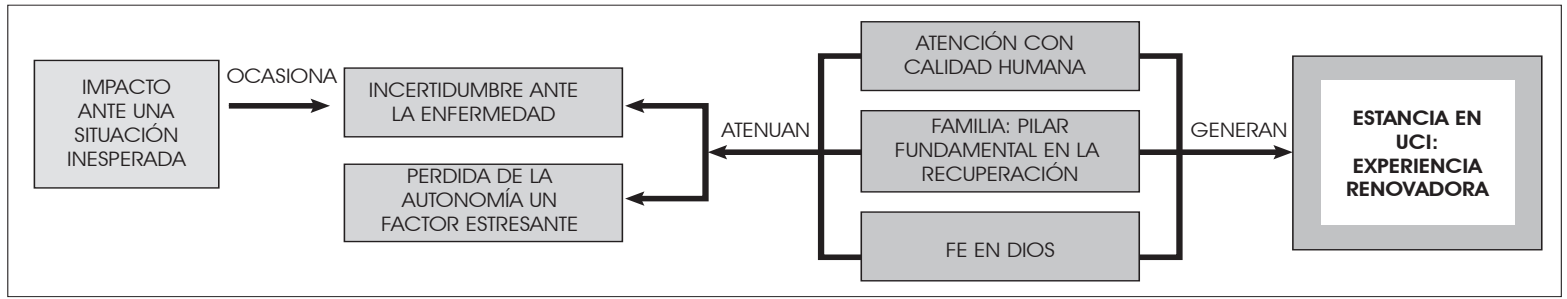

Figura 1. Categorías que representan las vivencias del paciente cardiovascular.

Fuente. Proceso de análisis de la investigación sobre vivencias del paciente cardiovascular durante la estancia en la unidad coronaria (2017).

\section{Estancia en la UCI: experiencia renovadora}

Para los participantes en el presente estudio de su estancia en la UCI se constituye en una experiencia renovadora, que genera un cambio realmente significativo para la vida del paciente. El haber sobrevivido a una vivencia cercana a la muerte, les causa gran alegría al considerar que es una nueva oportunidad para volver a vivir y para un nuevo comienzo.

I5E1: "Estoy muy contento, porque una oportunidad de volver a revivir, de volver, porque en el caso que me pasó, supuestamente yo ya era una muerte súbita".

Todo esto conlleva a pensar de una manera diferente; el paciente concibe su enfermedad como algo bueno, como un acontecimiento necesario para modificar su vida. Al mismo tiempo, experimentan cambios significativos y positivos, como aprender a valorar las pequeñas cosas de la vida que habían pasado por alto . Al mismo tiempo, quieren eliminar de sus vidas las actitudes negativas.

I2E1: "Mi vida cambió, mejoró, en el modo de pensar y hacer las cosas gracias a lo que pasé yo me renové completamente".

I4E2: "Lo que viví fue algo muy duro para mí, algo difícil, pero esto me hizo dar cuenta que puedo hacer más con mi vida, me ayudó a valorar más todo lo que tengo, me ayudó abrir los ojos".

Se inicia un periodo de gratitud con la vida y la nueva oportunidad que ésta les brinda, se percibe como una segunda oportunidad para vivir de una manera más saludable y positiva, se observa en ellos un resurgimiento por aprovechar al máximo la vida y hacerlo con más ganas.

\section{Fe en Dios}

En el presente estudio se advierte en los pacientes una necesidad de buscar a Dios para poder afrontar de una mejor manera esta vivencia. Esta fe se la percibe como el principal apoyo para superar, no solo el proceso de la enfermedad que están sufriendo, sino también cualquier acontecimiento negativo. El encomendarse a un ser supremo les da fortaleza para salir adelante y recuperarse, siendo también una fuente de esperanza.

I2E1: "Primero que todo yo me sentía acompañado muchísimo de mi Dios todopoderoso, esa es la parte principal".

I1E1 "Eso cuando a veces me sentía débil, como cuando empezaba a estar pensativa y me pasaba cosas malas por la cabeza de que no fuera a salir, de que no fuera a volver a la casa siempre, siempre me apoyaba en la oración, a Dios que le debo mucho, sin él no estaría aquí horita, le debo mi vida le debo todo".

Los participantes en el estudio se apoyan en Dios para afrontar las adversidades; con ayuda de su fe no ven su enfermedad como algo negativo que haya ocurrido en sus vidas, sino como los designios de Dios y lo interpretan como algo que tiene que pasar para darle sentido a sus vidas. 
ISSN-PRINT

1794-9831

E-ISSN 2322-7028

Vol. 16 No. 2

May - Ago 2019

Cúcuta, Colombia
También los informantes se aferran a su fe en Dios y la convierten en aceptación para afrontar los posibles resultados de su enfermedad; esta fe hace que quieran darle un significado al proceso de la enfermedad por el cual están atravesando, que no ocurrió por nada, sino por un propósito que Dios les ha dado.

I2E1: "Yo pienso que eso que me ocurrió es un propósito de Dios y es algo bueno, empezar una vida nueva".

I4E1: "Uno que más encomendarse a Dios porque él es el todo poderoso y es el que decide si uno se lo lleva o lo deja otro ratico más acá".

\section{Atención con calidad humana}

La atención con calidad humana por parte del equipo de salud, fue percibida por los entrevistados como un factor importante para afrontar y superar su proceso de enfermedad y los factores estresantes que encontraron en la unidad de cuidados intensivos. El equipo de salud, se caracterizó por crear un ambiente de aceptación y confianza y motivarlos permanentemente en su proceso de recuperación.

I1E1: "las muchachas también me decían, no hombre usted es un verraco, está bastante recuperado, se está recuperando bastante rápido, usted es una persona fuerte esperamos que siga así para que pueda salir rápido de acá".

Se puede observar que el personal de enfermería fue un apoyo constante para los entrevistados, tanto para querer luchar por su vida y no decaer, así como para aceptar los procedimientos que ellos mismo no conocían y que estaban dispuestos a realizar al sentirse respaldados por este mismo personal.

I3E1: "ah las enfermeras muy muy atentas eh ellas fueron unas de las personas que más me colaboraron para yo aceptar la cirugía en un momento estaba reacio".

Los entrevistados refirieron que la creación de un ambiente con un diálogo cálido de las personas que los cuidaban produjo en ellos un clima de confianza en sí mismos; también dijeron sentirse muy a gusto con la calidad de cuidado que percibieron en estas personas.

I4E1: "en general todas las que me atendieron a mi todos los días me saludaban, me daban los buenos días, me daban la comida cuando yo no tenía ganas de comer, me cambiaban el pañal, aunque tuviera pena".

En otro sentido, el cuidado humanizado y con un alto grado de calidad generó en los entrevistados sentimientos relacionados con la satisfacción de saber que alguien cuida de ellos.

I5E1: "Lo hacen a uno revivir en la forma en que lo atienden a uno allá, me dio mucha moral y muy bien atendido".

\section{Incertidumbre frente a la enfermedad}

En esta categoría, se logra observar cómo los informantes tienen mucho temor de lo que les pueda suceder mientras soportan su proceso de enfermedad, eso genera en ellos incertidumbre.

También se percibe que estos participantes al disponer de mucho tiempo libre en la unidad de cuidados intensivos, tenían pensamientos negativos sobre el proceso de la enfermedad, la mayoría de las veces por desconocimiento de la misma.

I1E1: "ahí, sentado, acostado, pero pues sin pensar, de que cuando a uno se le vienen como cosas negativas a la cabeza, de qué será, que, si salgo de ahí, será que, si salgo de esta, si me iré alentar".

Del mismo modo, los participantes en esta investigación refirieron sentir mucho temor y bastante incertidumbre frente al proceso de la enfermedad, debido a que tenían conocimientos muy escasos o nulos frente al problema que estaban enfrentando; además referían que les producía mucha incertidumbre la poca información que recibían del personal de salud acerca de su condición de salud.

I3E2: "uno lo llena como de incertidumbre no saber qué es lo que tiene uno ni que le van a hacer, porque aparte de que a uno casi no le dicen nada, cuando pregunta le dicen que espere que ya llaman a el doctor para que uno le explique y cuando viene el doctor pues a uno le habla todo raro y uno queda en las mismas entonces".

Otra cosa que generaba incertidumbre en los pacientes era el concepto que ellos tenían antes de entrar a la unidad de cuidados intensivos, que generalmente eran concepciones muy negativas respecto a la UCI que se la relaciona con la muerte o con invalidez; concluyendo que esto los predisponía causándoles un gran estrés. 
I4E1: "las personas que entran allá es que están muy graves o que se van a morir y pues uno también tenía ese concepto"

\section{Impacto ante una situación no esperada}

En esta categoría, se describe el impacto que se genera en los participantes al encontrarse frente a una situación no esperada. Los pacientes informaron sentir gran temor al enfrentarse a la enfermedad y refieren no sentirse a gusto ni aceptar la realidad, razón por la cual buscan motivos para salir de esa situación difícil.

I1E1: "uno no se ve en la situación como que me voy a morir uno nunca va aceptar esas cosas, claro que todos vamos para allá pero no quien va a querer morirse, uno se pone a pensar en todo lo que se deja mis hijos mi esposo *ay quien me hubiera cuidado a mi viejo* porque ya los hijos están grandes pero mi viejo es el que vive conmigo él está pa todo ahí al lado diuno y dejarlo solito me daba mucha tristeza".

Otro aspecto que es importante recalcar, es que los participantes refirieron sentir una gran preocupación ante la situación económica que causaban en el momento de ser internados en la unidad de cuidados intensivos, porque no tenían un trabajo estable al ser víctima de una enfermedad, razón por la cual no podrían responder económicamente por su familia

I6E1: "eso fue de verdad que me dejó muy pensativo, tras de que uno enfermo preocupándose que si sigue así de onde se va sacar plata, es que en estas condiciones uno no se puede dar el lujo de enfermarse, pues ya era duro lo que vivía y tocaba también pensar en la plata, de dónde íbamos a reunir para pagar lo que estaba allá, el tiempo que estuve allá por ese lado fue muy duro la salida fue costosa"

I6E1: "ese día que salí ni almorcé pensando en la plata que había que pagar y el afán que me daba con mi familia; a mí no me gusta depender de nadie, me acuerdo sí muy bien que no alcancé ni almorzar por eso, y eso fue rápido Virgen Santísima que yo me imaginaba todo ese platal y si pero de igual manera, pues como dice la plata pues no la tenían, pero ahí aunque sea de una forma u otra se consiguió".

\section{Familia: pilar fundamental en la recuperación}

En esta categoría se nombra a la familia como un pilar fundamental en la recuperación de los pacientes, ya que referían que en sus familiares encontraban una fuente de apoyo frente al proceso de la enfermedad. Además les daban ánimo para seguir adelante y para aceptar los procedimientos que de otra manera no hubieran aceptado; igualmente, sirvieron de conexión con los demás familiares que no podían ver.

I4E1: "Si claro, desde el primer día ellos estuvieron ahí, como dicen por ahí al pie del cañón, siempre pendiente de mí, siempre preguntando por mí, me llevaban mensajes de mis otros familiares que les escribían por el celular".

De igual manera, se percibe que la mayoría de los participantes manifiesta sentirse inconforme con el corto tiempo que se daba a las visitas de los familiares, porque para ellos es el único momento que tienen para pasar con sus seres queridos en el día y sentían que era muy reducido. Se puede deducir que para los pacientes es muy importante el tiempo que se les deja para las visitas.

I2E1: "pasan solamente a dos personas, o sea dos pero pasa una, digamos era una hora, media, una media hora uno, y media hora el otro, no más, eso pues si era como muy poquito tiempo, y uno como en el afán de hablar con ellos, se le pasa a uno esa horita volando, eso sí como que deberían cambiarlo"

Los participantes refirieron que a pesar de lo incómodo y estresante que resulta pasar por el proceso de la enfermedad, fue muy reconfortante saber que la familia siempre era un apoyo en todo momento, fue muy importante para ellos sentirse queridos por sus familiares a pesar de que hubiera diferencias antes de que sucediera dicha situación de enfermedad.

I2E1: "mis hijas ellas viven en Bogotá, y pa que le digo mentiras, yo con ellas no es que tenga una muy buena relación, lastimosamente, pero pues por lo que pase vinieron hasta aquí a verme después de cómo casi 7 años que no las veía y muy poco que hablamos, eso vinieron y me dieron mucha fuerza, me llenó mucho verlas y que sin importar la relación que, pues tenemos, vi que sea como sea ellas quieren mucho al taita, y me reconfortó mucho, pero mucho".

\section{Perdida de la autonomía: un factor estresante}

En esta categoría se describe la pérdida de la autonomía del paciente al estar en una situación crítica, lo cual genera un gran estrés al no poderse valer por sí mismos. Algunos participantes refirieron sentirse 
ISSN-PRINT

1794-9831

E-ISSN 2322-7028

Vol. 16 No. 2

May - Ago 2019

Cúcuta, Colombia muy incómodos con el hecho de no poder ir al baño por sí solos, ya que generalmente lo hacían sin ayuda, sentían que habían perdido su capacidad de valerse por sí mismos.

I3E1: "lo que si no me pude acomodar yo duré cuatro días en la UCI, después me hicieron la cirugía y duré un día más y me pasaron para piso, fue el hecho de tener que hacer uno las necesidades en la cama, eso es lo más incómodo que hay".

También los informantes señalaron que el ambiente en general (ruidos de los monitores, bombas de infusión, los equipos de soporte ventilatorios y los quejidos de los demás pacientes) en la unidad de cuidados intensivos fue un factor estresante que causó en ellos angustia y desesperación al encontrarse en un lugar que no les era familiar.

I1E1: "los ruidos de las maquinitas que eso que pitaban y que la otra gente que estaba allá cuando se quejaban y cosas, así como que lo perturban a uno".

I2E1: "yo sentía algo como que no podía hablar pues yo el tubo ese lo tenía en la boca, sentía como que me iba atorar, como que quería escupirlo; no, eso es una sensación terrible, que vaina más horrible, y que a uno lo tengan despierto, yo no sé porque es que no me sedaron si tenía esa vaina, y como también eso está conectado a un aparatico, eso me forzaba como a respirar, eso sí que no me gustó en lo absoluto"

\section{Discusión}

La vivencia de sufrir una enfermedad cardiovascular y su hospitalización en la UCI es difícil, debido a los sentimientos negativos que se generan como temor, ansiedad e incertidumbre; también genera angustia los efectos de la enfermedad como son: los equipos utilizados en el tratamiento, los procedimientos terapéuticos y los cuidados de bienestar y confort. Esto concuerda con los resultados encontrados en el estudio de Solano y González (7), el cual se describe que la experiencia de los pacientes es una vivencia que genera sentimientos como el miedo, el temor, la preocupación, la sensación de gravedad y la vivencia de una muerte cercana.

Esta vivencia, debido a su cercanía con la muerte y las situaciones estresantes que allí se generan, se percibe como dura. Como resultado de todas las vivencias experimentadas por los pacientes en la UCI suele producirse cambios positivos que repercuten en la vida de la persona.

Para Peplau (24), la enfermedad "Es una contribución a los procesos individuales de auto renovación, auto reparación y en la toma de conciencia hacia sí mismos y la propia vida." Esto se evidencia en los resultados presentados en este estudio, donde para los participantes su estancia en la UCI se constituye en una experiencia renovadora, que genera un cambio significativo para sus vidas, fomentándose una mayor gratitud por la nueva oportunidad que tienen de vivir, anhelando iniciar ciertas trasformaciones que les permitan ser mejores en todos los aspectos de su vida. Travelbee citado por Beltrán (25), afirma que transitar por una enfermedad grave "es una experiencia que cambia el ser humano, le permite avanzar en el entendimiento y en el autoconocimiento".

Del mismo modo, se vio reflejado que, desde el punto de vista espiritual, Dios fue un factor esencial para aceptar y darle algún tipo de sentido o propósito al proceso de enfermedad en el cual se encontraban.

Como pudo notarse en los resultados, la fe en Dios fue una explicación suficiente de los pacientes para salir adelante en su recuperación, potenciando sus ansias de vivir y así afrontar adecuadamente la situación en la que se encontraban. Esto mismo se evidencia en el estudio realizado por E. Santiago Castro quien dice que "el soporte espiritual del paciente está basado en sus creencias propias y religiosas como forma de disminuir y afrontar la situación de crisis que se está experimentando" (12).

Por otra parte, el escenario en el que se encuentran los participantes en este estudio, en donde se ven aislados de sus seres queridos, de su entorno y el estar en un ambiente desconocido en el cual se enfrentan con la realidad de estar críticamente enfermos, los lleva a apoyarse a un ser supremo, de ahí que se forme un lazo más fuerte con su religiosidad, conexión que se logra a través de la oración. Estos resultados se ven contrastados en el estudio de Collado (26) quien expone que "En esencia la espiritualidad es un fenómeno íntimo y trascendente que es vital para el desarrollo de la recuperación de calidad en el paciente. Además, una fortaleza, motivación y búsqueda del significado de la vida".

También es importante señalar, que el personal de salud juega un papel importante en el proceso de re- 
cuperación del paciente, siendo éste quien tiene el contacto directo con el enfermo la mayor parte del tiempo. El equipo de salud fue percibido como una ayuda irremplazable, en cuanto a la atención que le propiciaba y al apoyo que le aportaba, dándole ánimo en momentos de angustia, tristeza y soledad. Esto concuerda con lo dicho por Watson (27) para quien "el interés de la enfermería reside en comprender la salud como a la enfermedad y la experiencia humana". Igualmente, lo refiere el estudio realizado por Zambrano et al. (28) quienes mencionan que "para las informantes fue de vital importancia el papel del personal de enfermería en su recuperación en la (UCI), refieren que fue muy bueno y humanizado en todos los aspectos".

De modo similar, se descubrió en el estudio, que a los pacientes les generaba confianza el cuidado brindado por las enfermeras en la unidad al tener la sensación de estar vigilados y de percibir cierto lazo de familiaridad, lo cual les hace sentir seguros. Este resultado coincide con lo expresado por Watson (27) para quien "El grado de genuinidad y sinceridad de la expresión de la enfermera, se relaciona con la grandeza y eficacia del cuidado"; es decir, que la relación enfermeropaciente contribuye al bienestar de los usuarios--

No obstante, para el paciente el verse en la UCI, le generó mucha incertidumbre ante su enfermedad, principalmente por el desconocimiento acerca de su padecimiento y a la falta de información que le brindaba el personal médico; factores que concuerdan con lo dicho por Mishel (29) quien expresó que "La incertidumbre surge en el momento en que la persona no puede estructurar o categorizar adecuadamente los casos de enfermedad, debido a la falta de claves suficientes".

Este aspecto también se observa en el estudio realizado por Santiago et al., en donde se precisa que "Esta vivencia de estar hospitalizado en la UCI le implica al paciente un proceso de afrontamiento, que se caracteriza por una crisis dada por una situación para la que no se está preparado" en este caso el verse críticamente enfermos (12).

Algo semejante ocurre con la situación por la cual los pacientes se sienten mucho tiempo solos, sin comunicación alguna y sin ninguna distracción, lo que genera en ellos múltiples pensamientos negativos sobre su evolución; actitud que concuerda con lo dicho por
Mishel (29) "Cuanto más tiempo dure la incertidumbre en el contexto de la enfermedad, más inestable será el modo de funcionamiento del individuo aceptado previamente". Estos resultados también coinciden con lo encontrado en el estudio de Santiago et al. (12), en donde se dice que "El aislamiento, la falta de comunicación, separación de los familiares y de su vida laboral, todo esto genera en la persona sensaciones de soledad, abandono e incertidumbre, del no saber cuándo saldrá de allí, que procedimientos se le practicará y los posibles que sucederán".

De igual manera, es importante destacar que durante la vivencia del paciente en la unidad intensiva coronaria, la familia fue el pilar fundamental para lograr su recuperación, siendo este uno de los aspectos más importantes, debido a que al sentirse acompañado de un ser querido le genera tranquilidad. Esto se ve contrastado en la investigación realizada por Zambrano et al. (28), donde se ratifica que "La familia es la base fundamental de la sociedad, lo cual significa que para el paciente que está hospitalizado en la Unidad de Cuidados Intensivos (UCI), es primordial el apoyo y respaldo que reciban de estos para su proceso de afrontamiento de la enfermedad.

Así mismo, el apoyo que les brinda la familia a los pacientes genera en ellos ánimo, esperanza y motivación para salir pronto de la unidad. Igualmente, se evidencia que la enfermedad facilitó la unión de diferentes lazos familiares que se habían perdido. Lo cual coincide con lo dicho por Betty Neuman quien determinó que "la familia es vista como un subsistema de la sociedad, que es el sistema más importante" (20).

Dentro de este marco, es importante subrayar que las vivencias experimentadas en una UCI llegan a ser desconcertantes para los pacientes, debido a que es un entorno diferente al que ya están acostumbrados. En este espacio al principio les es difícil adaptarse porque no se sienten a gusto ni aceptan la realidad que están viviendo, generando sentimientos de temor. Este aspecto concuerda con lo mencionado en el estudio de Granberg et al. (13) el cual dice que las vivencias para estos pacientes son como "Una especie de estado de caos tras el inicio de su enfermedad, lesión o accidente, lo que dio lugar a sentimientos de extrema inestabilidad, vulnerabilidad y miedo, a menudo experimentan tensión interna prolongada".

Por otro lado, no todos los enfermos perciben negati- 
ISSN-PRINT

1794-9831

E-ISSN 2322-7028

Vol. 16 No. 2

May - Ago 2019

Cúcuta, Colombia vamente estas situaciones, sino que las asumen como una forma de recapacitar y cambiar los preconceptos que tenían sobre lo que les está sucediendo. Esto puede coincidir con lo postulado por Merle Mishel (29) quien afirma que "La adaptación representa la continuidad de la conducta biopsicosocial normal del individuo y constituye el deseado resultado de los esfuerzos del afrontamiento, bien para reducir el nivel de incertidumbre, vista como un peligro, bien para ver la incertidumbre como una oportunidad." Estudio que coincide con los resultados de la presente investigación, donde se ha podido deducir que el proceso de adaptación de cada paciente les ayudó a superar en cierta medida las situaciones novedosas a las que se enfrentaban.

Debe señalarse, que hay muchos factores estresantes cuando un paciente ingresa a la UCI, entre ellos está la pérdida de autonomía, debido a que la persona está acostumbrada a tener cierto grado de privacidad y a realizar las cosas por sus propios medios; por eso las afecta al tener que aceptar la ayuda de otras personas para que las suplan en la realización de ciertas necesidades. Este sentimiento se ve reflejado también en el estudio de Zambrano et al. (28), para quienes "Con relación a las actividades cotidianas, se proyecta la manera en la cual las personas están acostumbradas a trabajar o realizar sus diferentes actividades diarias, todo esto depende del nivel socioeconómico y cultural al que pertenezca el individuo, todo esto se ve afectado y es extrañado por los pacientes en el nuevo mundo en el cual no tienen ni pueden hacer nada de lo que realizan habitualmente"; lo que genera en ellos el pensamiento de volverse una carga para quienes lo rodean.

En resumidas cuentas y como lo especifica Callista Roy, el entorno se define como "Todas las situaciones, circunstancias e influencias susceptibles de afectar el desarrollo y comportamiento de las personas o grupos" (30), definición que concuerda con la presente investigación, donde se deduce que los pacientes manifiestan que el entorno en la UCI es desconocido, poco familiar, donde se presentan situaciones con las que no están cómodos, causando en ellos sentimientos de angustia y desesperación.

\section{Conclusiones}

- La hospitalización en una Unidad de Cuidado Intensivo Coronario, es una vivencia estresante y dolorosa para los pacientes; situación que se puede atenuar con la atención con calidad humana ofrecidopor parte del personal de salud, el apoyo familiar como pilar fundamental en la recuperación y la fe en Dios. Todo esto, convierte la hospitalización en la UCI en una vivencia significativa que marca la vida de estas personas.

- Un aspecto que resalta de esta vivencia, son los sentimientos que se generaron en los pacientes como el temor ante su enfermedad, la incertidumbre debido a la falta de información sobre su situación, el estrés por los procedimientos que se les realizaban, la ansiedad que experimentaban ante el hecho de querer salir rápidamente de la UCI; factores que sobrellevaron mediante la atención que les brindó el personal de salud generando en ellos esperanza y confianza en su pronta recuperación. También les sirvió de gran ayuda el apoyarse en sus creencias, ya que esta actitud les brindó fortaleza espiritual para aceptar esta experiencia de una manera positiva. Otro hecho que los motivó a salir adelante fue el apoyo familiar que produjo en ellos gratitud, al saber que se preocupaban por su salud y recuperación, así como la alegría de saber que no estaban solos en esta situación.

- La vivencia más significativa ante una enfermedad crítica y el haber estado hospitalizado en una unidad de cuidados intensivos coronarios, fue percibida como una experiencia renovadora. Este suceso cercano a la muerte produjo cambios positivos en la forma de ver y enfrentar la vida, puesto que lo percibieron como un nuevo comienzo, dejando a un lado múltiples actitudes negativas, que afectaban tanto su vida personal como social.

\section{Conflicto de Intereses}

Los autores declaran no tener ningún conflicto de intereses. 


\section{Referencias bibliográficas}

1. Organización Mundial de la Salud. Enfermedades cardiovasculares. [Internet] Datos y cifras 2017 [Citado el 09/03/17] Encontrado en: http://www.who.int/mediacentre/factsheets/fs317/es/

2. Ministerio de la protección social. Guía para manejo de urgencias. Tomo I. [Internet] 2009. [citado 09/03/17]. Disponible en: http://www.minsalud.gov.co/documentos\%20y\%20publicaciones/ gu $\%$ c3\%ada $\% 20$ para $\% 20$ manejo $\% 20 \mathrm{de} \% 20$ urgencias $\% 20$-tomo $\% 20$ i.pdf

3. Texas Heart Institute. Factores de riesgo cardiovascular. [Internet] 2016. [citado el 09/03/17] Disponible en: http://www.texasheart.org/HIC/Topics_Esp/HSmart/riskspan.cfm

4. Ministerio de salud y protección social. Análisis de situación de salud (ASIS) Colombia, Bogotá, noviembre de 2016. [Citado el 12/06/18] Disponible en: https:/www.minsalud.gov.co/sites/rid/Lists/ BibliotecaDigital/RIDE/VS/ED/PSP/asis-colombia-2016.pdf

5. Ministerio de Salud. Colombia enfrenta epidemia de enfermedades cardiovasculares y diabetes. Boletín de prensa No. 077 de 2014. [Citado el 09/03/17] Disponible en: https://www.minsalud.gov.co/ Paginas/Colombia-enfrenta-epidemia-de-enfermedades-cardiovasculares-y-diabetes.aspx

6. Ministerio de Salud. Análisis de Situación de Salud con el Modelo de Determinantes Sociales Departamento Norte de Santander 2016. [Citado el 12/06/18]. Disponible en: http://salasituacionalidsnds. weebly.com/uploads/1/0/7/1/10714324/asis-departamental-2016-nortedesantander.pdf

7. Solano Ruiz M, Siles González J. Las vivencias del paciente coronario en la unidad de cuidados críticos. Index Enferm [Internet]. 2005 [citado 2017-03-09]; 14(51):29-33. Disponible en: http://scielo. isciii.es/scielo.php?script $=$ sci arttext\&pid $=\mathrm{S} 1132-12962005000300006 \& \operatorname{lng}=\mathrm{es} \& \mathrm{nrm}=\mathrm{iso}>. \quad \mathrm{ISSN}$ 1132-1296.

8. Guitierrez B, Blanco Alvariño A, Luque Perez M, Ramirez Perez M. Experiencias, percepciones y necesidades en la UCI: Revision sistematica de estudios cualitativos. Enfermeria Global [Internet]. 2007. [Citado 04/09/17] Disponible en: http://revistas.um.es/eglobal/article/view/822/842

9. Stayt L. Death, empathy and self-preservation: the emotional labour of caring for families of the critically ill in adult intensive care. Intensive nursing [Internet]. U.S. 2012. [Citado 04/09/17]; 18(9):126775 Disponible en: https://www.ncbi.nlm.nih.gov/pubmed/19413555

10. Rivera Alvarez L. El cuidado al paciente cardiovascular en situaciones que generan un gran sentimiento de pérdida, amenaza, temor o muerte. Av. Enferm [Internet]. 2008 [Citado 04/09/17]; 26(1):124133. Disponible en: http://www.revistas.unal.edu.co/index.php/avenferm/article/view/12892/13652

11. Hofhuis JG, Spronk PE, van Stel HF, Schrijvers AJ, Rommes JH, Bakker J. Experiences of critically ill patients in the ICU. Intensive and Critical Care Nursing [Internet]. 2008 [citado 07/09/17]; 24(5):30013. Disponible en: https://www.ncbi.nlm.nih.gov/pubmed/18472265

12. Santiago de Castro E, Vargas Rosero E. Experiencia de estar hospitalizado en una unidad de cuidado intensivo coronario de Barranquilla. Av. Enferm [Internet]. 2015. [Citado 04/09/17]; 33(3):381-390. Disponible en: http://www.scielo.org.co/pdf/aven/v33n3/v33n3a06.pdf

13. Granberg A, Bergbom I, Lundberg D. Patients' experience of being critically ill or severely injured and cared for in an intensive care unit in relation to the ICU syndrome. Intensive and Critical Care Nursing [Internet]. 1998 [Citado 04/09/17]; 14:294-307. Disponible en: https://www.ncbi.nlm.nih. gov/pubmed/10196913

14. Hughes F, Bryan K, Robbins I. Relatives' experiences of critical care. Nurs Crit Care [Internet]. 2005 [Citado 04/09/17]; 10(1):23-30. Disponible en: https://www.ncbi.nlm.nih.gov/pubmed/15739636

15. Del Barrio M, Lacunza M, Armendáriz A. pacientes con transplante hepático: su experiencia en cuidado intensivo. Estudio fenomenológico. Enfermería Intensiva [Internet]. 2001 [citado 14/09/17]; 12(3):109-161. Disponible en: https://doi.org/10.1016/S1130-2399(01)78031-5

16. Gómez Londoño E. El cuidado de enfermería del paciente en estado crítico: Una perspectiva bioética. 
ISSN-PRINT

$1794-9831$

E-ISSN 2322-7028

Vol. 16 No. 2

May - Ago 2019

Cúcuta, Colombia
Persona y Bioética [Internet]. 2008 [citado 19/09/17]; 12(2):145-157. Disponible en: http://personaybioetica.unisabana.edu.co/index.php/personaybioetica/article/view/966/1307

17. Madjar I. Infligir y Manejar el dolor: un desafío para la Enfermería. Invest Educ Enferm [Internet]. 2000 [citado 03/09/17]; 18(1):37-47. Disponible en: http://www.redalyc.org/pdf/1052/105218294003. pdf

18. Rodríguez Gómez L. La investigación cualitativa, libro virtual [Citado el 16 de octubre del 2017] Disponible en: http://www.esup.edu.pe/division_investigacion_material.html

19. Briones G. Metodología de la investigación cuantitativa en las ciencias sociales. [online]. Bogotá, ARFO Editores e Impresores Ltda. [Citado el 11 octubre 2017] Disponible en: ftp://ftp.puce.edu.ec/ Facultades/CienciasEducacion/Maestria/CienciasEducacion/Paralelo1/modulo2.pdf

20. Ulin $P$, Robinson E, Tolley E. Investigación aplicada en salud pública Métodos cualitativos [Online]. Washington, DC; 2006. [citado 1 noviembre de 2017] Disponible en : http://iris.paho.org/xmlui/bitstream/handle/123456789/729/9275316147.pdf? sequence $=1$

21. Castillo E, Vásquez M. El rigor metodológico en la investigación cualitativa. Colombia Médica [Internet]. 2003 [Citado el 28 de octubre del 2017]; 34(3):164-167. Disponible en: http://www.redalyc. org/pdf/283/28334309.pdf

22. Rada D. El rigor en la investigacion cualitativa: Técnicas de análisis, credibilidad, transferibilidad y confirmabilidad. Revista Venezolana de Investigación [Internet]. 2007 [Citado el 28 de octubre del 2017]. Disponible en: www.capacidad.es/sica09/Comunicaciones/C202\%20Credibilidad.doc

23. Informe de Belmont. 1979. U.S.A. 2015. [Citado el 28 de octubre del 2017]. Disponible en: http:// www.bioeticayderecho.ub.edu/archivos/norm/InformeBelmont.pdf

24. Marron Machaca N. Relación terapéutica enfermera - paciente según la teoría. Hildegard E. Peplau en los servicios de cirugía, del Hospital Regional Manuel Núñez Butrón - Puno 2013. [Internet] Perú, 2014. [Citado 23/08/17]. Disponible en: http://repositorio.unap.edu.pe/handle/UNAP/2249

25. Beltrán Salazar OA. La experiencia de estar hospitalizado en una unidad de cuidado intensivo. [Tesis maestría]. Universidad de Antioquia; 2007. Disponible en: http://bibliotecadigital.udea.edu.co/dspace/ bitstream/10495/153/1/AriasMaria_2007_SignificadoExperienciaCriticamenteEnfermo.pdf

26. Collado RE. Cuidado espiritual, labor del profesional de Enfermería [revista en Internet]. 2010 [citado: 2 sep 2017]. Disponible en: en: http://cremc.ponce.inter.edu/360/revista360/ciencia/Cuidado\%20 espiritual\%20enfermeria.pdf

27. Watson Harman J. "Love and Caring: Ethics of Face and Hand-an Invitation to Return to the Heart and Soul of Nursing and our Deep Humanity“. Nursing Administration Quarterly. [Internet] 2010. [citado 10/10/17]. Disponible en: https://www.nursingcenter.com/journalarticle?Article $\underline{\mathrm{ID}}=447370$ \&Journal_ID $=54011 \&$ Issue ID $=447363$

28. Zambrano Plata G, Ferreira Cardona J, Lindarte Clavijo A, Niño Bayona C, Ramírez Rodríguez N, Rojas Bautista L. Entre la incertidumbre y la esperanza: Percepciones y motivaciones de los pacientes hospitalizados en la unidad de cuidados intensivos (UCI). Rev. cienc. cuidad. [Online]. 2016 [Citado 15 Febrero 201]; 7(1):52-60 Disponible en: http://revistas.ufps.edu.co/ojs/index.php/cienciaycuidado/ article/view/493

29. Mishel M. The university of North Carolina at Chapel Hill, Teoría de la incertidumbre frente a la enfermedad. [Citado 15 Febrero 2017] Disponible en: http://teoriasintermedias2013.blogspot.com. co/2013/06/merle-h-mishel-teoria-de-la.html

30. Alligood M, Tomey A. Modelos y teorías de enfermería, séptima edición. Teoría de la Adaptación de Callista Roy. 